\title{
Assessment of the TEM Information Limit by Means of Tilted Illumination
}

\author{
Heiko Müller, Stephan Uhlemann, Joachim Zach, Peter Hartel, and Max. Haider
}

\section{Corrected Electron Optical Systems GmbH, Heidelberg, Germany}

Aberration correctors for the spherical aberration $C_{s}$ [1] and more recently also for the chromatic aberration $C_{c}[2,3]$ have improved the capabilities of the transmission electron microscope (TEM) for quantitative atomic-resolution imaging. Since aberration correctors help to adjust imaging conditions for optimum contrast, finally the information limit set by incoherent effects has become the most important parameter in the design of novel instrumentation. Due to the partial coherent nature of the imaging process each TEM image has to be understood as an incoherent sum over a series of sub-images, slightly displaced in lateral or longitudinal direction. The effect of the lateral displacement we call image spread while that of the longitudinal is called focus spread. Aberration correctors can reduce the negative impact of the finite convergence angle $\sigma\left(\vartheta_{\mathrm{sc}}\right)$ and of the finite energy width in the illumination on the information transfer [3]. Nevertheless, image and focus spread with standard deviation $\sigma\left(A_{0}\right)$ and $\sigma\left(C_{1}\right)$ due to other effects remain and must be quantified.

Imaging of ultra-thin amorphous foils (e.g. tungsten, carbon) under tilted illumination conditions is the standard method for aberration measurement and alignment in TEM [5]. It has already been pointed out $[6,7]$ that the corresponding diffractograms also provide information about the information limit. Under the assumption of linear phase contrast with scattering amplitude $F(\vec{g})=F^{\star}(-\vec{g})$ the diffractogram of an amorphous specimen with illumination tilt $\vec{\tau}$ can be calculated analytically from the transmission cross-coefficient $T_{\mathrm{cc}}=T_{\mathrm{cc}}\left(\vec{g}, \vec{g}^{\prime}\right)$ in reciprocal space [4]

$$
I(\vec{g})=F(\vec{g})\left|T_{\mathrm{cc}}(\vec{\tau}+\vec{g}, \vec{\tau})-T_{\mathrm{cc}}(\vec{\tau}, \vec{\tau}-\vec{g})\right|
$$

Owing to the rotational symmetry of the optical system all damping effects related to the primary aberrations $C_{c}$ and $C_{s}$ vanish on circles of radius $|\vec{\tau}|$ about the tilt centres at $\pm \vec{\tau}$ if the defocus is appropriately adjusted to $C_{1}=-|\lambda \vec{\tau}|^{2} C_{s}$. Along these so-called achromatic circles the single-side-band contrast mechanism sets the intensity amplitude to $I(\vec{g})=1 / 2 F(\vec{g})$ if no image spread is present. The radial width of the circles in reciprocal space then is determined by

$$
\sigma(|\vec{g}|)=\frac{1}{2 \pi \lambda} \sqrt{\left(\frac{1}{\sigma\left(C_{1}\right)|\vec{\tau}|}\right)^{2}+\left(\frac{1}{2 \sigma\left(\vartheta_{\mathrm{sc}}\right) C_{s} \lambda|\vec{\tau}|^{2}}\right)^{2}}
$$

Figs. 1 compares calculated and measured diffractograms taken without aberration corrector. Under typical conditions the width of the circles is primarily determined by $1 /\left(\sigma\left(\vartheta_{\mathrm{sc}}\right) C_{s}\right)$. True achromatic circles can be observed in a $C_{s}$-corrected TEM.

Scanning along the circles as shown in Fig. 1 extracts unique information about the image spread. To match the measurement with the simulations the scattering amplitude and the modulation transfer function of the camera have to be taken into account. This method is useful to compare image spread between different instruments or different alignments of the beam path. Experimentally, some care is necessary since parasitic astigmatism and axial coma or residual dispersion in the untilted situation can lead to erroneous results. Also asymmetric ring patterns can be observed in such cases. For optimum contrast on the circles the intentionally adjusted defocus and the tilt-induced astigmatism must fulfill the relation $C_{1}=-\left|A_{1}\right|$. This can readily be observed from the Thon ring pattern at lower spatial 
frequencies. If high-tension centre and rotation centre do not coincide sufficiently well with the aligned coma-free centre the mismatch will cause additional image spread, but this would not be an artefact of the measurement but a real defect of the optical instrument under characterization.

We will demonstrate that the assessment of focus and image spread by means of tilted illumination is a versatile and practical tool to investigate and optimize the information limit of modern TEM instruments.

References:

[1] M. Haider et al, Nature 292 (1998) p. 768.

[2] P. Hartel et al, Proc. 14th EMC, Aachen, Germany (2008) p. 27.

[3] M. Haider et al, Microsc. Microanal. 16 (2010) p. 393.

[4] K. Ishizuka, Ultramicroscopy 5 (1980) p. 55.

[5] S. Uhlemann and M. Haider, Ultramicroscopy 72 (1998) p. 109.

[6] C. Boothroyd and R. Dunin-Borkowski, Proc. of 16th IMC, Sapporo, Japan (2006) p. 640.

[7] J. Barthel and A. Thust, Phys. Rev. Lett. 101 (2008) 200801.
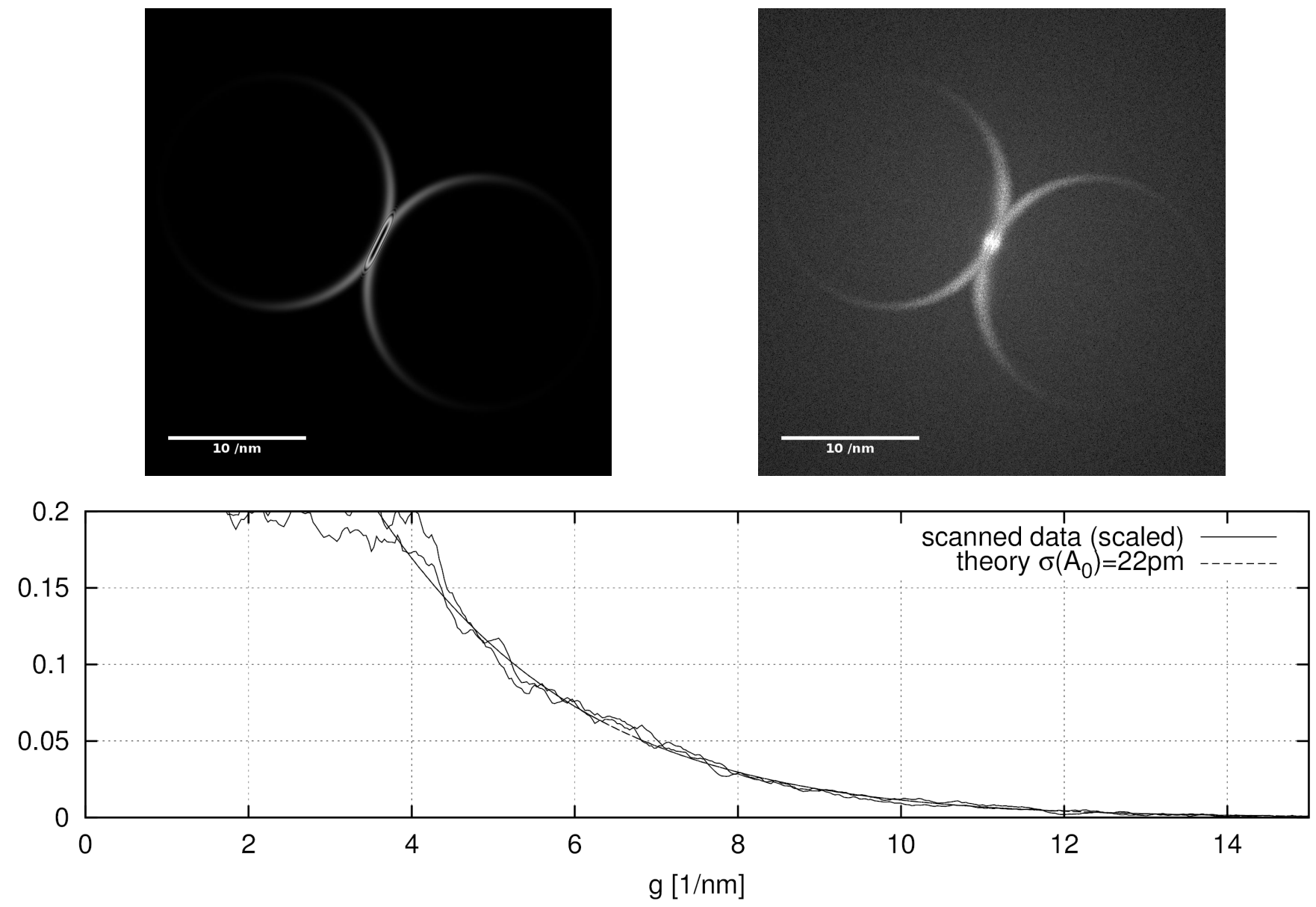

Figure 1. Simulated (left) and recorded (right) diffractograms with an illumination tilt of $2^{\circ}$. At the bottom scans along the two branches of the achromatic circles corresponding to different directions are compared with the contrast expected for an isotropic image spread of $\sigma\left(A_{0}\right)=22 \mathrm{pm}$. 